\title{
Conjugate Phase MRI Reconstruction With Spatially Variant Sample Density Correction
}

\author{
Douglas C. Noll*, Member, IEEE, Jeffrey A. Fessler, Senior Member, IEEE, and Bradley P. Sutton, Member, IEEE
}

\begin{abstract}
A new image reconstruction method to correct for the effects of magnetic field inhomogeneity in non-Cartesian sampled magnetic resonance imaging (MRI) is proposed. The conjugate phase reconstruction method, which corrects for phase accumulation due to applied gradients and magnetic field inhomogeneity, has been commonly used for this case. This can lead to incomplete correction, in part, due to the presence of gradients in the field inhomogeneity function. Based on local distortions to the $k$-space trajectory from these gradients, a spatially variant sample density compensation function is introduced as part of the conjugate phase reconstruction. This method was applied to both simulated and experimental spiral imaging data and shown to produce more accurate image reconstructions. Two approaches for fast implementation that allow the use of fast Fourier transforms are also described. The proposed method is shown to produce fast and accurate image reconstructions for spiral sampled MRI.
\end{abstract}

Index Terms - Image reconstruction, magnetic field inhomogeneity, magnetic resonance imaging, spiral imaging.

\section{INTRODUCTION}

$\mathbf{I}$ MAGE reconstruction in conventional magnetic resonance imaging (MRI) is usually performed via an inverse Fourier transform of samples acquired in k-space (Fourier domain). This can be accomplished using a simple discrete Fourier transform if the samples lie on a Cartesian grid and if the underlying static magnetic fields are essentially uniform across space. This paper examines image reconstruction for the case where these conditions do not hold. The conventional approach for image reconstruction of non-Cartesian sampled MRI is the conjugate phase reconstruction method, as originally proposed by Macovski [1]. This was extended in [2], [3] to include correction for magnetic field inhomogeneities and explicit expressions for sample density correction. Image reconstruction without compensation for the density variation can result in a severely degraded point spread function. Determination of the density compensation term has been an active topic for investigation with analytical [2], [4], [5] and numerical approaches [6], [7]. Common

Manuscript received August 30, 2004; November 15, 2004. This work was supported in part by the National Institutes of Health (NIH) under Grant R01 DA015410. The Associate Editor responsible for coordinating the review of this paper and recommending its publication was X. Hu. Asterisk indicates corresponding author.

*D. C. Noll is with the Department of Biomedical Engineering, University of Michigan, 1107 Carl A. Gerstacker, 2200 Bonisteel Blvd, Ann Arbor, MI 48109-2099 USA (e-mail: dnoll@umich.edu).

J. A. Fessler is with the Department of Electrical Engineering and Computer Science, University of Michigan, Ann Arbor, MI 48109-2122 USA.

B. P. Sutton is with the Biomedical Imaging Center, Beckman Institute, University of Illinois at Urbana-Champaign, Urbana, IL 61801 USA.

Digital Object Identifier 10.1109/TMI.2004.842452 to all of these approaches is that the density compensation function (DCF) depends only on the time or k-space location allowing this correction to be implemented by a simple scaling of the acquired data. Over the years, there has been substantial progress in developing computationally efficient approaches to the conjugate phase reconstruction, for example convolution gridding interpolation [8], [9] to map the samples to a Cartesian grid, thus allowing the use of the fast Fourier transform (FFT) for Fourier inversion. Noll et al. [10], [11] have demonstrated fast algorithms for implementation of the conjugate phase reconstruction that combine with time or frequency approximations to the inhomogeneity-related phase accumulation terms. For Cartesian sampled MRI like spin-warp imaging or echoplanar imaging (EPI), sample density correction is generally not necessary and magnetic field inhomogeneities can lead to geometric and intensity distortions. There is an extensive body of literature on correction of these distortions; one of the more common approaches is the pixel shift method [12], [13].

The conjugate phase $(\mathrm{CP})$ reconstruction and most of the spin-warp and EPI correction methods assume the integrity of the k-space trajectory. That is, images are reconstructed based on the assumption that the underlying static magnetic fields do not affect the k-space sample locations. However, any smoothly varying inhomogeneity function will have in-plane gradients that vary spatially. This results in a distorted k-space trajectory, which also varies spatially. In other words, each point in space has its own k-space trajectory and this needs to be accounted for in the image reconstruction. The concept of local k-space has been proposed previously in several imaging application including echo-planar imaging [14] and spiral imaging [15], [16]. These works noted changes in sensitivity, apparent echo time, and signal loss resulting from in-plane gradients.

In this paper, we observe that the spatially variant distortions in k-space trajectory change the overall pattern of the acquisition of spatial frequency information and also the density of samples within that pattern. This causes both shape and intensity distortions to the point-spread function, which can lead to blurring or intensity variations in the resultant images. We propose a modification to the conjugate phase reconstruction in which the DCF depends on both time and space for objects with spatially variant magnetic field inhomogeneity. This approach is shown to correct for these distortions to the point-spread function. We also demonstrate that the same concept can be used to explain and correct for intensity variations in EPI and spin-warp imaging. Finally, we investigate rapid algorithms to implement or closely approximate the spatially variant conjugate phase reconstruction. 


\section{THEORY}

This theory section starts with an analysis of the effects of in-plane gradients in one-dimension (1-D). The results here are directly applicable to distortions and their correction in EPI and spin-warp imaging. Next, we derive the conjugate phase (CP) reconstruction method for 2-D spiral imaging with explicit expressions for the DCF. Following that, we analyze linear and local gradients that distort the k-space trajectory and modify the DCF. The net result is a modified CP reconstruction with spatially variant DCF. Finally, we introduce a fast approximation to the proposed method.

\section{A. Conjugate Phase Reconstruction in One Dimension With Inhomogeneity}

We first examine a 1-D frequency encoding pulse sequence with a constant gradient $G$. For $g=(\gamma / 2 \pi) G$, the gradient in units of frequency/distance (e.g., $\mathrm{Hz} / \mathrm{cm})$ and $\Delta f$, the inhomogeneity function, the signal equation is

$$
s(t)=\int m(x) \exp (-i 2 \pi(\Delta f(x)+g x) t) d x .
$$

We now define a coordinate transformation operator

$$
x_{d}=S[x]=x+\frac{\Delta f(x)}{g}
$$

where $S[\cdot]$ is the operator that maps true object locations to distorted image locations. Assuming that $(d / d x) \Delta f(x)+g=$ $\Delta f^{\prime}(x)+g>0$, the frequency $f(x)=\Delta f(x)+g x$ is a monotonically increasing function of $x$ and the transformation operator of (2) is invertible: $x=S^{-1}\left[x_{d}\right]$. A typical value of $\mathrm{g}$ for an EPI pulse sequence is $100 \mathrm{~Hz} / \mathrm{cm}$ and in the experimental data below, the peak derivative is $\pm 50 \mathrm{~Hz} / \mathrm{cm}$, so the condition is often valid in practice. Note that the inverse transformation may be numerically unstable when the peak derivative is close in magnitude to the peak gradient values.

Making a substitution of variables for $x_{d}$ and using $d x_{d}=$ $\left(1+\Delta f^{\prime}(x) / g\right) d x$, we rewrite the signal equation as

$$
\begin{aligned}
s(t) & =\int \frac{m\left(S^{-1}\left[x_{d}\right]\right)}{1+\frac{\Delta f^{\prime}\left(S^{-1}\left[x_{d}\right]\right)}{g}} \exp \left(-i 2 \pi k(t) x_{d}\right) d x_{d} \\
& =\int m_{d}\left(x_{d}\right) \exp \left(-i 2 \pi k(t) x_{d}\right) d x_{d}=M_{d}(k(t))
\end{aligned}
$$

where $k(t)=\int_{0}^{t} g d \tau=g t$ is the $\mathrm{k}$-space location, and $m_{d}$ and $M_{d}$ are the distorted object and its Fourier transform. A standard Fourier reconstruction yields

$$
\hat{m}(x)=F^{-1}\left\{M_{d}(k)\right\}=m_{d}(x)=\frac{m\left(S^{-1}[x]\right)}{1+\frac{\Delta f^{\prime}\left(S^{-1}[x]\right)}{g}}
$$

which is the original object with both a coordinate (geometric) distortion $S^{-1}[x]$ and an intensity distortion $\left(1+\Delta f^{\prime}\left(S^{-1}[x]\right) / g\right)^{-1}$.

From this equation, we can easily see that the desired image can be obtained by spatial shifts [12], [13] and correcting for intensity variations. To determine $m\left(x_{0}\right)$, we merely need to look in the distorted image, $\hat{m}$, at location $x=S\left[x_{0}\right]$ and then adjust the image intensity.

We now examine the $\mathrm{CP}$ reconstruction in this context. For this approach, we define $\left(g+\Delta f^{\prime}(x)\right)$ as a "local sample density" correction term that varies as a function of space rather than the usual case where it varies as a function of $k$ or time. The $\mathrm{CP}$ reconstruction is then

$\hat{m}_{c p}(x)=\left(g+\Delta f^{\prime}(x)\right) \int s(t) \exp (i 2 \pi[\Delta f(x)+g x] t) d t$.

By substituting $k(t)=g t, d k=g d t$ and $s(t)=M_{d}(k(t))$, this expression can be written as

$$
\begin{aligned}
\hat{m}_{c p}(x) & =\left(1+\frac{\Delta f^{\prime}(x)}{g}\right) \int M_{d}(k) \exp (i 2 \pi k S[x]) d k \\
& =\left(1+\frac{\Delta f^{\prime}(x)}{g}\right) m_{d}(S[x])=m(x) .
\end{aligned}
$$

In one dimension, the $\mathrm{CP}$ reconstruction can be thought of as a Fourier domain interpolation equivalent to the pixel shift method. Note that for these implementations, both the pixel shift method and conjugate phase reconstruction method require the undistorted field map and its derivative. There are other pixel shift methods that use distorted field maps [17] and other reconstruction approaches, such as the simulated phase evolution rewinding (SPHERE) method [18], that use distorted field maps and do not require its derivative.

\section{B. Image Reconstruction for Two-Dimensional (2-D) Spiral Imaging}

The method developed in Section II-D, below, is applicable to a variety of trajectories; however, for concreteness we focus on the CP method and the DCF for spiral imaging. In 2-D, the signal equation without magnetic field inhomogeneity is

$$
s(t)=\iint m(\mathbf{x}) \exp (-i 2 \pi \mathbf{k}(t) \cdot \mathbf{x}) d \mathbf{x}=M(\mathbf{k}(t))
$$

where $\mathbf{k}(t)$ is the spiral $\mathbf{k}$-space trajectory. We parameterize $\mathbf{k}(t)$ in a manner similar to radial $\mathrm{k}$-space imaging, that is, as a function of time and an idealized continuum of starting phase angles, $\phi$, which can represent shot number. For this case, the CP method [2], [5] is

$$
\begin{aligned}
\hat{m}(\mathbf{x}) & =\iint M(\mathbf{k}) \exp (i 2 \pi \mathbf{k} \cdot \mathbf{x}) d \mathbf{k} \\
& =\iint s(t) \exp (i 2 \pi \mathbf{k}(t) \cdot \mathbf{x})\left|\frac{\partial\left(k_{x}, k_{y}\right)}{\partial(t, \phi)}\right| d t d \phi
\end{aligned}
$$

where $\left|\partial\left(k_{x}, k_{y}\right) / \partial(t, \phi)\right|$ is the determinant of the Jacobian of the transformation from $(t, \phi)$ to $\left(k_{x}, k_{y}\right)$. Hoge et al. [5] noted that this Jacobian, which matches the areas of integration in the two coordinate systems, is a sample DCF. If the Jacobian is nonsingular, then $\hat{m}(\mathbf{x})=m(\mathbf{x})$.

The determinant of the Jacobian of this transformation is

$$
\begin{aligned}
D(t) & =\left|\begin{array}{cc}
\frac{\partial k_{x}}{\partial t} & \frac{\partial k_{x}}{\partial \phi} \\
\frac{\partial k_{y}}{\partial t} & \frac{\partial k_{y}}{\partial \phi}
\end{array}\right|=\left|\begin{array}{cc}
g_{x}(t) & -k_{y}(t) \\
g_{y}(t) & k_{x}(t)
\end{array}\right| \\
& =\mathbf{g}(t) \cdot \mathbf{k}(t) \\
& =|\mathbf{g}(t)||\mathbf{k}(t)| \cos (\angle \mathbf{g}(t)-\angle \mathbf{k}(t))
\end{aligned}
$$


where $g=(\gamma / 2 \pi) G$ is the gradient in $\mathrm{Hz} / \mathrm{cm}$. This expression contains 1) the outward velocity of the k-trajectory, $|\mathbf{g}(t)| \cos (\angle \mathbf{g}(t)-\angle \mathbf{k}(t))$, times 2$)$ the $\mathrm{k}$-space radius, $|\mathbf{k}(t)|$. Hoge et al. [5] postulated that these two terms represent 1) speed away from origin and 2) the $|\rho|$ weighting terms appropriate for radial sampling strategies (e.g., projection imaging). For the special case of an Archimedean spiral, the expressions in (9) can also be shown to be equivalent to another DCF derived by Meyer et al. [4] (contrary to an assertion in [5]). For the Archimedean spiral parameterized as

$$
\mathbf{k}(t)=k_{x}(t)+i k_{y}(t)=A \varphi(t) \exp (i(\varphi(t)+\phi))
$$

where $\varphi(t)$ is the angular position versus time function and $A=$ $N_{s} /(2 \pi \cdot F O V)$ where $N_{s}$ is the number of shots, the DCF simplifies to Meyer's expression

$$
D(t)=A|\mathbf{g}(t)| \sin (\angle \mathbf{g}(t)-\angle \mathbf{k}(t)) \text {. }
$$

This expression is often better behaved numerically, particularly for large k-space values. In many spiral trajectories, the sample density approaches a constant for large $|\mathbf{k}|$ values and (9) relies on approximate cancellation of large $|\mathbf{k}|$ and a small outward velocity, which is very sensitive to numerical errors in calculating the latter. The expression in (9), however, is valid for non-Archimedean spirals such as variable density spirals.

\section{Image Reconstruction for 2-D Spiral Imaging for Linearly Distorted Trajectories}

As a prelude to the shift variant case, in this section, we modify the DCF to accommodate a spiral trajectory distorted by a linear gradient. Irarrazabal, et al. [19] proposed an image reconstruction approach where the inhomogeneity function can be approximated by an offset frequency and a constant background gradient

$$
\Delta f(\mathbf{x})=\Delta f_{0}+\mathbf{g}_{b} \cdot \mathbf{x}
$$

where

$$
\mathbf{g}_{b}=\nabla \Delta f(\mathbf{x})=\left[\begin{array}{c}
\frac{\partial \Delta f(x, y)}{\partial x} \\
\frac{\partial \Delta f(x, y)}{\partial y}
\end{array}\right]=\left[\begin{array}{l}
g_{x b} \\
g_{y b}
\end{array}\right] .
$$

Combining this gradient with the applied gradient yields a distorted k-space trajectory

$$
\mathbf{k}_{d}(t)=\left[\begin{array}{l}
A \varphi(t) \cos (\varphi(t)+\phi)+g_{x b} t \\
A \varphi(t) \sin (\varphi(t)+\phi)+g_{y b} t
\end{array}\right]
$$

and the signal equation is

$$
\begin{aligned}
s(t) & =\exp \left(-i 2 \pi \Delta f_{0} t\right) \iint m(\mathbf{x}) \exp \left(-i 2 \pi \mathbf{k}_{d} \cdot \mathbf{x}\right) d \mathbf{x} \\
& =\exp \left(-i 2 \pi \Delta f_{0} t\right) s_{d}(t)
\end{aligned}
$$

where $s_{d}(t)$ is the signal evaluated on the distorted trajectory.
The Jacobian determinant for the transformation from $(t, \phi)$ to $\left(k_{x 0}, k_{y 0}\right)$ is

$$
\begin{aligned}
D^{\prime}(t)=\left(\mathbf{g}(t)+\mathbf{g}_{b}\right) \cdot \mathbf{k}(t) \\
=|\mathbf{k}(t)|(|\mathbf{g}(t)| \cos (\angle \mathbf{g}(t)-\angle \mathbf{k}(t)) \\
\left.\quad+\left|\mathbf{g}_{b}\right| \cos \left(\angle \mathbf{g}_{b}-\angle \mathbf{k}(t)\right)\right) .
\end{aligned}
$$

Here, the outward velocity term is modified by the background gradient. The second half of this expression is analogous to the $\left(g+\Delta f^{\prime}(x)\right)$ density correction term in the 1-D case. When the background gradient is in the same direction of the $\mathrm{k}$-space location, the gradient pushes the trajectories farther apart, requiring an increase in the sample density correction in an amount proportional to $\left|\mathrm{g}_{b}\right|$. The CP method is possible if the Jacobian determinant is positive, so we require that

$$
\frac{d|\mathbf{k}(t)|}{d t}>\left|\mathbf{g}_{b}\right|
$$

i.e., the outward velocity of the k-space trajectory must be larger than the background gradient.

An alternate expression for the Jacobian determinant is

$$
\begin{aligned}
D^{\prime}(t)= & A|\mathbf{g}(t)| \sin (\angle \mathbf{g}(t)-\angle \mathbf{k}(t)) \\
& +\left|\mathbf{g}_{b}\right||\mathbf{k}(t)| \cos \left(\angle \mathbf{g}_{b}-\angle \mathbf{k}(t)\right) \\
= & D(t)+D_{b}(t) .
\end{aligned}
$$

Using the Jacobian determinant corrects for variations in sample density due to the background gradients, whereas Irarrazabal, et al. [19] used a postgridding density compensation approach. Fig. 1 shows a standard k-space trajectory and its DCF as well as two distorted trajectories and their DCFs.

The proposed CP image reconstruction method for this case will be

$$
\begin{aligned}
\hat{m}(\mathbf{x})= & \iint s_{d}(t) \exp \left(i 2 \pi \mathbf{k}_{d}(t) \cdot \mathbf{x}\right) D^{\prime}(t) d t d \phi \\
= & \iint s(t) \exp (i 2 \pi \Delta f(\mathbf{x}) t) \\
& \times \exp (i 2 \pi \mathbf{k}(t) \cdot \mathbf{x}) D^{\prime}(t) d t d \phi .
\end{aligned}
$$

If the condition (17) is satisfied, this is an exact reconstruction. The last expression in (19) looks exactly like the CP reconstruction with off-resonance corrections, except that the DCF, $D^{\prime}(t)$, is different.

\section{Image Reconstruction for 2-D Spiral Imaging for Spatially Varying Inhomogeneity}

We now extend the above analysis to allow for a spatially variant off-resonance function $\Delta f(\mathbf{x})$ with a nonconstant background gradient

$$
\mathbf{g}_{b}(\mathbf{x})=\nabla \Delta f(\mathbf{x})=\left[\begin{array}{l}
\frac{\partial \Delta f(x, y)}{\partial x} \\
\frac{\partial \Delta f(x, y)}{\partial y}
\end{array}\right]=\left[\begin{array}{l}
g_{x b}(x, y) \\
g_{y b}(x, y)
\end{array}\right] .
$$



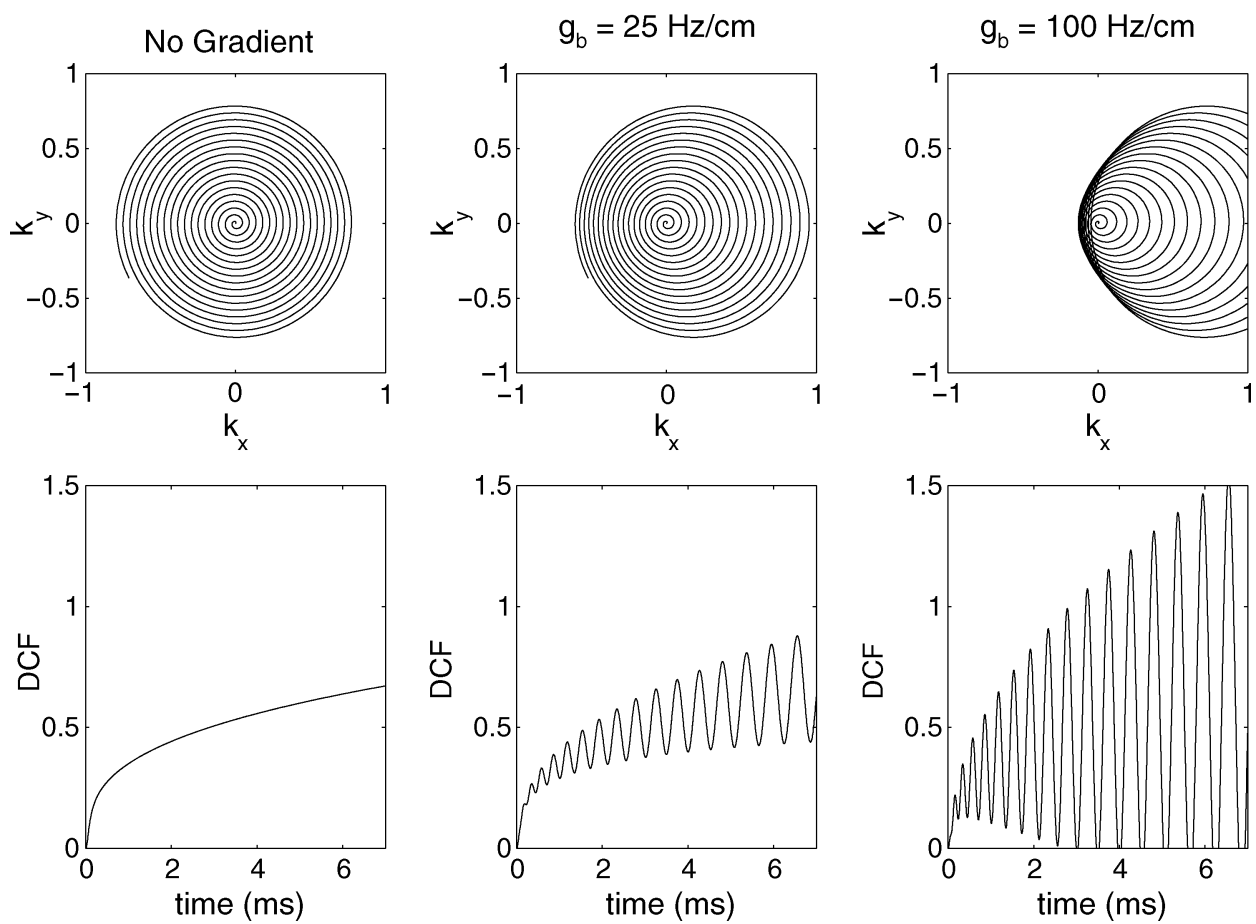

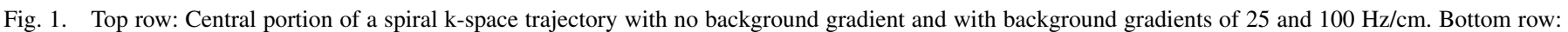

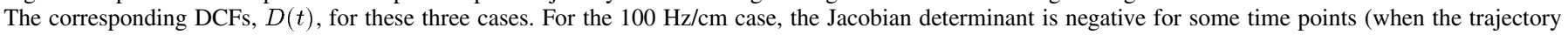
crosses over itself).

We propose that the $\mathrm{CP}$ reconstruction with spatially variant density compensation be

$\hat{m}(\mathbf{x})=\iint s(t) \exp (i 2 \pi \Delta f(\mathbf{x}) t) \exp (i 2 \pi \mathbf{k}(t) \cdot \mathbf{x}) D(\mathbf{x}, t) d t d \phi$

where $D(\mathbf{x}, t)$ is a spatially variant DCF based on the Jacobian determinant, for example

$$
\begin{aligned}
D(\mathbf{x}, t)= & A|\mathbf{g}(t)| \sin (\angle \mathbf{g}(t)-\angle \mathbf{k}(t)) \\
& +\left|\mathbf{g}_{b}(\mathbf{x})\right||\mathbf{k}(t)| \cos \left(\angle \mathbf{g}_{b}(\mathbf{x})-\angle \mathbf{k}(t)\right) \\
= & D(t)+D_{b}(\mathbf{x}, t) .
\end{aligned}
$$

Here, $D(t)$ is the standard density correction term and $D_{b}(\mathbf{x}, t)$ is the sample density adjustment due to the background gradients. The criteria for the nonnegativity of the Jacobian determinant is now

$$
\frac{d|\mathbf{k}(t)|}{d t}>\left|\mathbf{g}_{b}(\mathbf{x})\right|
$$

for all $t$ and $\mathbf{x}$. It is possible that this condition may not be satisfied only for particular points in space and particular samples in time. One strategy for addressing this situation is to set the DCF equal to zero for these combinations of spatial locations and k-space samples, resulting in these samples being excluded from the image reconstruction.

Another interpretation of the condition in (23) is that when a point is reconstructed at a location $\mathbf{x}_{0}$, no other voxel locations will blur onto that location. In 1-D imaging with a constant gradient, the off-resonance induced spatial shift is $\Delta f(x) / g$. In this case, we argue that the distorted PSF will not shift from $x$ to $x_{0}$ if the frequency difference between these points divided by the gradient is less than the difference between spatial locations of these points (e.g., $\left|\Delta f(x)-\Delta f\left(x_{0}\right)\right| / g<\left|x-x_{0}\right|$ ). In spiral imaging, the gradient vector points in all directions and the shift/blur from the smallest gradient will dominate. The smallest gradient is the outwards (radial) gradient, and, thus, we argue that the criterion for which the center of the point spread function (PSF) will not blur from $\mathbf{x}$ to $\mathbf{x}_{0}$ is

$$
\frac{\left|\Delta f(\mathbf{x})-\Delta f\left(\mathbf{x}_{0}\right)\right|}{\min \frac{d|\mathbf{k}(t)|}{d t}}<\left|\mathbf{x}-\mathbf{x}_{0}\right|
$$

for all $\mathbf{x}$. Thus,

$$
\frac{\left|\Delta f(\mathbf{x})-\Delta f\left(\mathbf{x}_{n}\right)\right|}{\left|\mathbf{x}-\mathbf{x}_{n}\right|} \leq \max \left|\mathbf{g}_{b}(\mathbf{x})\right|<\min \frac{d|\mathbf{k}(t)|}{d t}
$$

which is the same condition as (23). This is a formal description of smoothness necessary for the $\mathrm{CP}$ reconstruction, which has been described heuristically by Schomberg [20].

\section{E. Fast Algorithms}

The image reconstruction method in (21) cannot be implemented directly using gridding and FFTs [8], [9]. In Noll, et al. [10], [11], fast algorithms were proposed based on segmentation of the off-resonance term, $\exp (i 2 \pi \Delta f(\mathbf{x}) t)$, along either the time or frequency dimensions. These approaches implement the standard $\mathrm{CP}$ reconstruction that uses only the spatially invariant density compensation term, $D(t)$

$\hat{m}(\mathbf{x})=\iint s(t) \exp (i 2 \pi \Delta f(\mathbf{x}) t) \exp (i 2 \pi \mathbf{k}(t) \cdot \mathbf{x}) D(t) d t d \phi$. 
We briefly summarize the time-segmented approach, in which (26) can be approximated as

$$
\begin{array}{r}
\hat{m}(\mathbf{x})=\iint s(t)\left[\sum_{l=0}^{L-1} a(t-l \tau) \exp (i 2 \pi \Delta f(\mathbf{x}) l \tau)\right] \\
\times \exp (i 2 \pi \mathbf{k}(t) \cdot \mathbf{x}) D(t) d t d \phi
\end{array}
$$

where $L$ and $\tau$ are the number and spacing of time segments, respectively. The terms in the square brackets approximate the complex exponential in time and $a(\cdot)$ can be thought of as an interpolation kernel that interpolates between samples of the exponential at times $l \tau$. This equation can be rewritten as

$$
\begin{aligned}
\hat{m}(\mathbf{x}) & =\sum_{l=0}^{L-1} \exp (i 2 \pi \Delta f(\mathbf{x}) l \tau) \\
\times & {\left[\iint s(t) a(t-l \tau) \exp (i 2 \pi \mathbf{k}(t) \cdot \mathbf{x}) D(t) d t d \phi\right] }
\end{aligned}
$$

where the terms in the square brackets represent the reconstruction of the signal $s(t) a(t-l \tau)$. This expression can now be implemented rapidly using gridding and FFTs. Accordingly, this conjugate phase reconstruction can be implemented with $L$ FFTs and the accuracy of the reconstruction is controlled by the number of segments, $L$. Sutton, et al. [21], derive optimal interpolation kernels and also evaluate error versus number of segments for a variety of different field maps.

Unfortunately, the temporal-spatial variability of the $D_{b}(\mathbf{x}, t)$ term in (21) prevents the direct use of this fast algorithm. Brute force implementation would require approximately $O\left(N^{4}\right)$ operations, where $N$ is the image matrix size along one-dimension. By comparison, the time- or frequency-segmented reconstruction [10], [11] requires approximately $O\left(L N^{2} \log N\right)$ operations. This difference can be substantial, particularly for large images like $N=256$, where the difference in reconstruction time is approximately three orders of magnitude (e.g., several seconds versus one hour).

We propose two methods for rapid implementation of (21). For the first method, we start by rewriting (22) as

$$
D(\mathbf{x}, t)=D(t)+g_{x b}(\mathbf{x}) k_{x}(t)+g_{y b}(\mathbf{x}) k_{y}(t)
$$

which can be implemented rapidly as the weighted sum of three time-segmented gridding reconstructions, for example

$$
\begin{aligned}
\hat{m}(\mathbf{x})= & \iint s(t) \exp (i 2 \pi \Delta f(\mathbf{x}) t) \\
& \times \exp (i 2 \pi \mathbf{k}(t) \cdot \mathbf{x}) D(t) d t d \phi \\
& +g_{x b}(\mathbf{x}) \iint s(t) \exp (i 2 \pi \Delta f(\mathbf{x}) t) \\
& \times \exp (i 2 \pi \mathbf{k}(t) \cdot \mathbf{x}) k_{x}(t) d t d \phi \\
& +g_{y b}(\mathbf{x}) \iint s(t) \exp (i 2 \pi \Delta f(\mathbf{x}) t) \\
& \times \exp (i 2 \pi \mathbf{k}(t) \cdot \mathbf{x}) k_{y}(t) d t d \phi .
\end{aligned}
$$

In essence, the reconstruction takes the result of the reconstruction with the invariant density compensation [the first line of (30)] and adds to it the corrections for the $\mathrm{x}$ and $\mathrm{y}$ background gradients (the second and third lines, respectively). One issue for this density compensation term is how to handle the situation where the maximum background gradient condition is violated.
When this occurs, the Jacobian determinant becomes negative and in general, one would set $D_{b}(\mathbf{x}, t)$ to zero to eliminate particular data samples from the image reconstruction for particular locations in space. This can be done for the $O\left(N^{4}\right)$ reconstruction, but cannot be done for the fast algorithm described here. In practice, we have found that the differences between these two approaches are imperceptible as they affect only a small number of $\mathrm{k}$-space samples for a small number of points in space. This reconstruction can, therefore, be implemented as $O\left(3 L N^{2} \log N\right)$, achieving substantial computational savings.

A second approximation to the reconstruction in (21) is suitable for use in gradient echo (GRE) imaging. In GRE, a background gradient leads to a shifted k-space trajectory and thus, there is different sample density for the origin in k-space (unless the background gradient is so large that the origin is not acquired at all). Here, a simple correction is to adjust the intensity of the reconstructed values from a standard density compensated reconstruction by a multiplicative correction factor. To do this, the DCF is evaluated at a single time point

$$
\begin{aligned}
\tau(x, y) & =\arg _{t}\left(\mathbf{g}_{b}(x, y)(T E+t)+\mathbf{k}(t)=0\right) \\
& =\arg _{t}\left(\left|\mathbf{g}_{b}(x, y)(T E+t)\right|=|\mathbf{k}(t)|\right)
\end{aligned}
$$

that is, the time $\tau$ where the shifted $\mathrm{k}$-space trajectory crosses the origin. At this time point, $\mathbf{g}_{b}$ and $\mathbf{k}$ point in either the same or opposite directions for typical spiral-in [22] and spiral-out trajectories acquisitions, respectively. Thus, the cosine term in (22) is 1 or -1 , respectively, and the correction term is

$$
\begin{aligned}
C(x, y) & =\frac{D(\tau(x, y))+D_{b}(x, y, \tau(x, y))}{D(\tau(x, y))} \\
& =\frac{D(\tau(x, y)) \pm\left|\mathbf{g}_{b}(x, y)\right| \cdot|\mathbf{k}(\tau(x, y))|}{D(\tau(x, y))}
\end{aligned}
$$

( + and - for the spiral-in and spiral-out trajectories, respectively). This is illustrated graphically in Fig. 2 for a spiral-out acquisition with a $\mathrm{TE}=20 \mathrm{~ms}$ and a background gradient of 25 $\mathrm{Hz} / \mathrm{cm}$. Observe that the k-space trajectory is shifted toward positive $k_{x}$ positions and the point where the trajectory crosses the origin in k-space is sampled with higher density. The spatially variant DCF is reduced relative to the invariant DCF and the intensity correction factor, $C$, is the ratio of these values. This correction, therefore, will reduce the intensity variations and allow the use of a fast reconstruction, but this does not correct for distortion in the PSF. In this approach, the conjugate phase reconstruction with inhomogeneity correction is modified with a point-by-point correction factor, $C(x, y)$. This reconstruction can be implemented as $O\left(L N^{2} \log N\right)$ and also achieves a substantial computational savings over the reconstruction in (21).

\section{METHODS}

\section{A. Simulation Study}

To evaluate the effectiveness of the proposed spatially variant density compensation, we performed two simulation studies. In the first, a circular object of radius $2 \mathrm{~cm}$ was simulated for no background gradient and for a constant background gradient of $g_{x}=25 \mathrm{~Hz} / \mathrm{cm}$. The object was reconstructed using the conjugate phase reconstruction using DCF functions $D(t), D^{\prime}(t)$, and using the postreconstruction intensity compensation. We also 


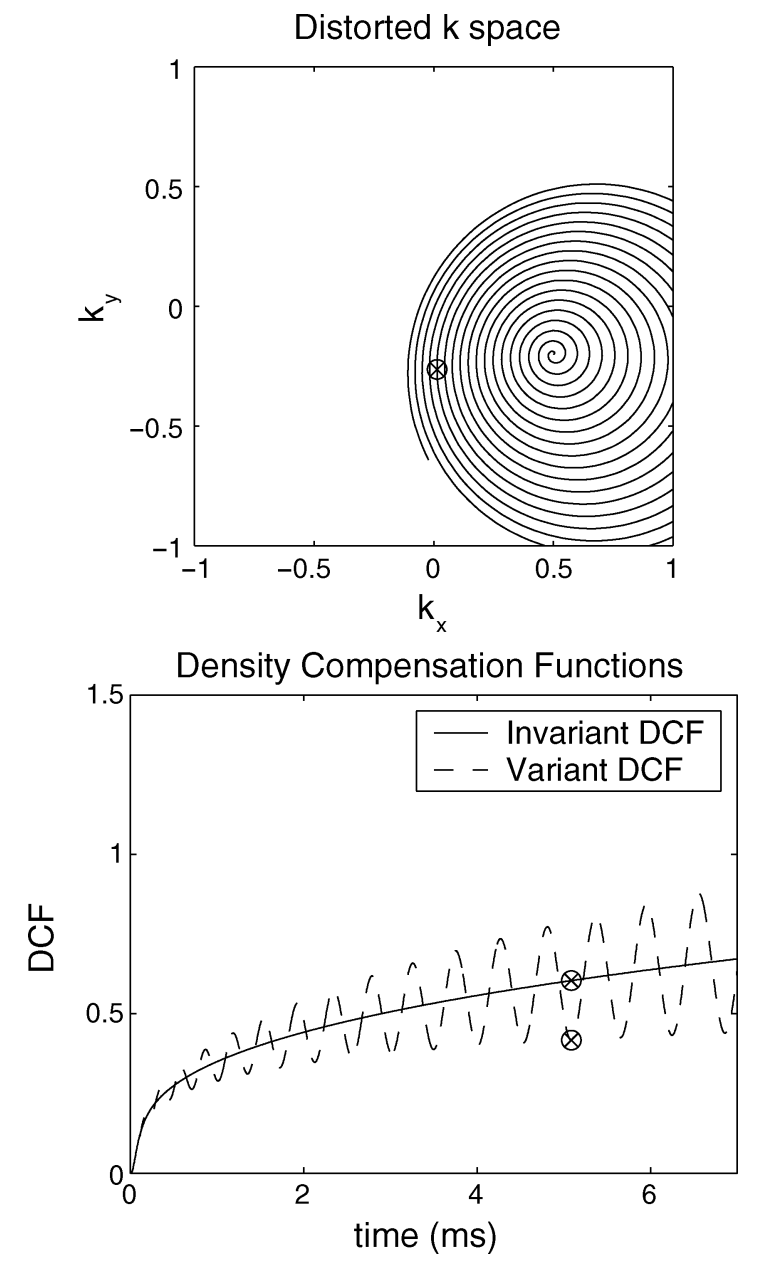

Fig. 2. Top: The central portion of a spiral-out k-space trajectory with a background gradient of $25 \mathrm{~Hz} / \mathrm{cm}$ and a gradient echo $\mathrm{TE}=20 \mathrm{~ms}$. The origin of k-space is denoted by $\otimes$. Bottom: The spatially invariant and variant DCFs for this trajectory with values at the time corresponding to crossing of the k-space origin denoted by $\otimes$.

simulated the effects of errors in the inhomogeneity function used for image reconstruction, including errors in $\Delta f$ and in the parallel $\left(g_{x}\right)$ and orthogonal $\left(g_{y}\right)$ gradient terms.

In the second simulation study, spiral data were simulated using a high-resolution simulation model of the human head. This model was derived from imaging data acquired on a $3.0 \mathrm{~T}$ GE Signa scanner using a three-dimensional (3-D) acquisition using two different echo times (spoiled GRE pulse, TE $=9$ and $10 \mathrm{~ms}, \mathrm{TR}=33$, Flip angle $=25, \mathrm{FOV}=16.5 \times 22 \mathrm{~cm}$, matrix $=192 \times 256$, slice thickness $=1 \mathrm{~mm}$, and 128 slices). Images were reconstructed off-line preserving the phase information, and the magnetic field (resonant frequency) map was determined from the phase difference [23] using $\Delta \omega(x, y)=\Delta \phi(x, y) / \Delta T E$. The selection of $\Delta T E=1 \mathrm{~ms}$ prevented $2 \pi$ phase jumps in the phase maps. Fat suppression was used to eliminate jumps in the frequency map associated with water/fat boundaries. To reduce noise in the frequency map, we filtered $\Delta \omega(x, y, z)$ by smoothing this map with a 3-D Gaussian kernel with width $\sigma=3.2 *$ (pixel size). To estimate a smooth field map for the slice of interest, we used the conjugate gradient algorithm to minimize a quadratic cost function consisting of a weighted norm term (based on the magnitude image) and a quadratic roughness penalty. This particular smoothing approach was chosen because it smoothly extends the field map over gaps in the image and into the background. The simulated signal samples were calculated using

$$
\begin{aligned}
s_{n}= & s\left(t_{n}\right) \\
= & \sum_{x, y} m(x, y) \exp \left(-i 2 \pi\left(k_{x}\left(t_{n}\right) x+k_{y}\left(t_{n}\right) y\right)\right. \\
& \times \exp \left(-i \Delta \omega(x, y)\left(t_{n}+T E\right)\right)
\end{aligned}
$$

where $m(x, y)$ is the high-resolution imaging data set (from $\mathrm{TE}=9 \mathrm{~ms})$ and $t_{n}$ is the time relative to the echo time associated with sample $s_{n}$ (e.g., for the spiral-in trajectory, where samples occur before the TE, $t_{n}$ will be negative). The magnetization image, $m(x, y)$ was thresholded to eliminate background noise from the simulation. $\mathrm{T}_{2}$ decay was not included in the simulation.

The simulated spiral acquisitions used a field of view of $22 \mathrm{~cm}$ and covered a k-space area of diameter 64 using 4617 data samples. The simulated sample period was $4 \mu$ s for a total readout duration of $18.46 \mathrm{~ms}$. The outward velocity $d|\mathbf{k}| / d t$ varied from $341 \mathrm{~Hz} / \mathrm{cm}$ to $53 \mathrm{~Hz} / \mathrm{cm}$. The spiral-out (forward) data used a simulated TE $=20 \mathrm{~ms}$ and spiral-in (reverse) used a simulated $\mathrm{TE}=30 \mathrm{~ms}$. The following reconstruction methods were performed for both simulated spiral-out and spiral-in acquisitions:

1) the direct Fourier reconstruction in (8) without inhomogeneity correction;

2) the CP reconstruction in (19) using only the spatially invariant DCF term, $D(t)$, implemented with the fast timesegmented approximation;

3) the $\mathrm{CP}$ reconstruction in (21) using the combined spatially variant DCF, $D(\mathbf{x}, t)$, implemented using a brute force summation;

4) the fast approximation to 3) described in (30), again implemented with the fast time-segmented approximation;

5) the reconstruction of part 2) using spatially invariant DCF with the postreconstruction intensity correction of (32);

6) the SPHERE method [18];

7) the iterative conjugate gradient [21] using a small number of iterations.

The fast time-segmented gridding approximation to the CP reconstruction used gridding with interpolation kernels of width four Cartesian samples to map the samples to a two-times oversampled Cartesian grid, and used $L=5$ time segments with min-max optimized temporal interpolators of Sutton et al. [21]. In addition to differences resulting from fast implementation of the conjugate phase reconstruction, 3) and 4) also differ in the handling of the case of a negative Jacobian determinant. For the approach in 3), samples where the Jacobian is negative are excluded from the reconstruction whereas in 4), they are included using the negative weighting. For reconstruction methods 3)-5), the gradients necessary for spatially variant image reconstructions were calculated from the inhomogeneity map using $3 \times$ 3 and $5 \times 5$ derivative kernels, the latter being less sensitive to noise in the field map.

In addition to variants of the $\mathrm{CP}$ reconstruction, for comparison, we have also implemented two other approaches to correct for magnetic field inhomogeneity. The first of these, the SPHERE method [18], takes the distorted image and field map 

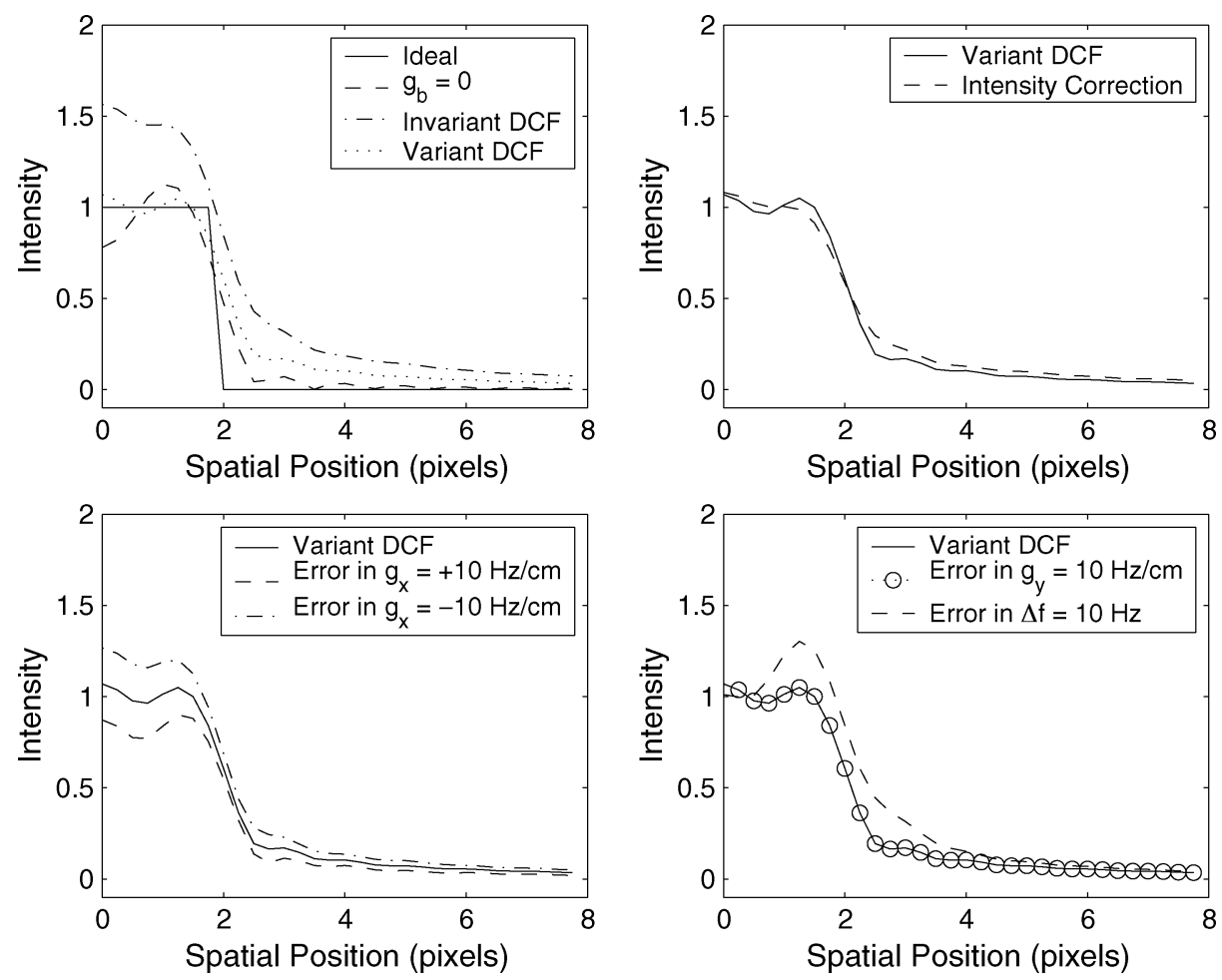

Fig. 3. Top left: Profiles along $x$ direction for the reconstruction of a simulated circular object of radius 2 pixels for no background gradient and for an $x$ direction background gradient of $25 \mathrm{~Hz} / \mathrm{cm}$ and a gradient echo with $\mathrm{TE}=20 \mathrm{~ms}$. The spatially invariant DCF produces overestimated the image intensity. Top right: comparison of response of spatially variant DCF to the invariant DCF response with intensity scaled by the ratio of DCF values in Fig. 2 . Bottom left: Effect of error in estimated $x$ gradient. Bottom right: effect of error in the estimated $y$ gradient and magnetic field strength.

and creates a synthetic $\mathrm{k}$-space data set using the negative inhomogeneity function. This synthetic data set is then reconstructed to produce a corrected image. The main idea is to exactly undo phase effects from magnetic field inhomogeneity and requires that the field maps lie in the same frame as the distorted image. For some applications this is a potential advantage, but for our simulations the distorted field maps were generated from two simulated images with different echo times and then smoothed using a $3 \times 3$ kernel to remove small ripples in the calculated map. Fast time-segmented approaches are possible, though they were not used in this implementation.

The second non-CP approach is the iterative conjugate gradient approach of Sutton et al. [21]. This approach also simulates the raw data from an estimated object, but using the undistorted field map (rather than the negative and distorted maps in SPHERE). Conceptually, this can be seen as simulating the physics of the MRI acquisition and then differences between the measured and simulated raw data drives updates to the estimated image. This approach is particularly interesting since the first iteration is the same as the CP reconstruction [21], [24] if the image is initialized to zeros and the data are weighted by the DCF. The DCF weighting, however, is statistically suboptimal, so we follow the approach suggested in [21] by using the DCF weighting for only the first iteration and uniform data weighting for all subsequent iterations. The iterative reconstruction method was implemented using the fast time-segmented approximations. With this implementation, two iterations of the iterative reconstruction will use approximately the same number of computations as the reconstruction in 4) Ten iterations were performed, and the images were analyzed after each iteration. The regularization parameter for this work was set to a value of 4, which produced a PSF with a full-width half maximum of 1.15 pixels for both spiral-in and spiral-out.

All images from the different reconstruction methods were compared to a "gold standard" reference image using a normalized root-mean-square error (NRMSE), where the errors were normalized by the RMS value of the reference image. The reference image was created by simulating raw data and reconstructing the same head slice with a standard Fourier reconstruction, but with the inhomogeneity term, $\Delta \omega$, set equal to zero.

The sensitivity of each reconstruction method to noise in the field map as well as noise in the raw data was also tested. To evaluate the effect of noise in the field map, 20 realizations of white Gaussian noise with standard deviations varying from 0 to $5 \mathrm{~Hz}$ were added to the field map used in the reconstruction. For cases where a gradient must be calculated, we also used the noisy field maps. The NRMSE was calculated with respect to the no noise case for each reconstruction and plotted versus standard deviation. This was examined for nonlinear behavior and the slope of the NRMSE versus standard deviation line was determined. To evaluate the effect of noise in the raw data, 20 realizations of bi-variate white Gaussian noise were added to the simulated raw data with standard deviations that were selected to produce signal-to-noise ratios that varied from 0 to approximately 10. Again, the NRMSE versus standard deviation plots were examined for nonlinear behavior and the slopes of these lines were calculated.

\section{B. Experimental Study}

Spiral data were acquired on a human volunteer subject using a single-shot, gradient echo spiral-out acquisition with 


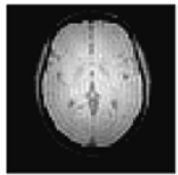

(a)

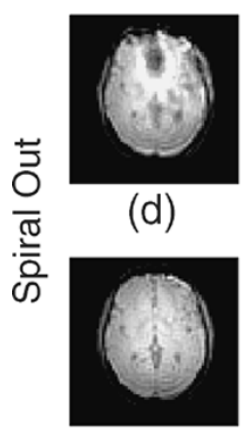

(h)

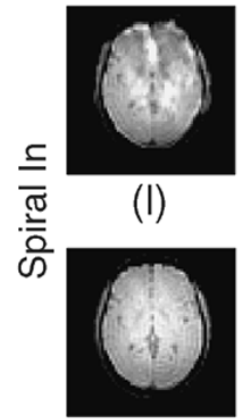

(p)

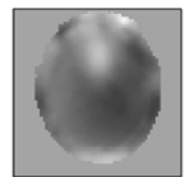

(b)

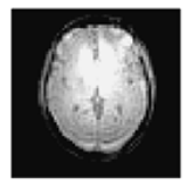

(e)

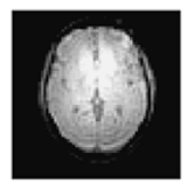

(i)

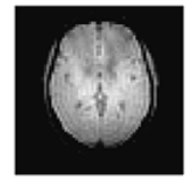

(m)

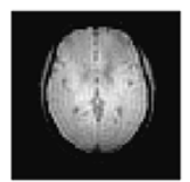

(q)
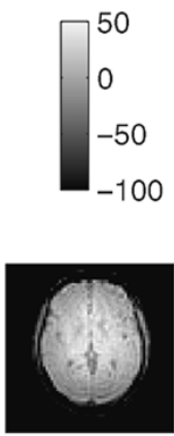

(f)

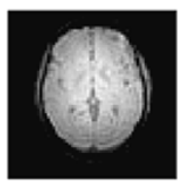

(j)

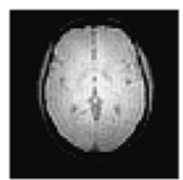

(n)

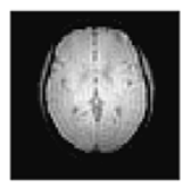

(r)

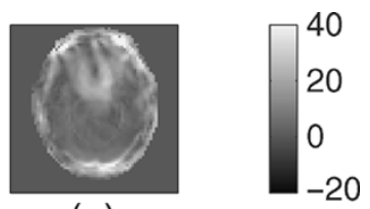

(c)

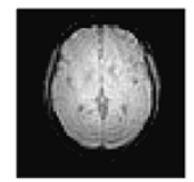

(g)

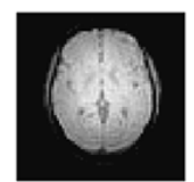

(k)

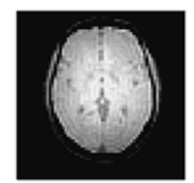

(o)

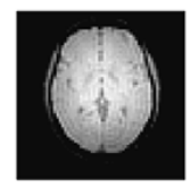

(s)

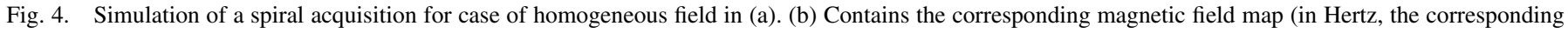

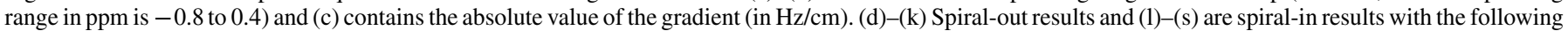

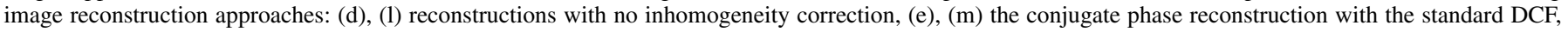

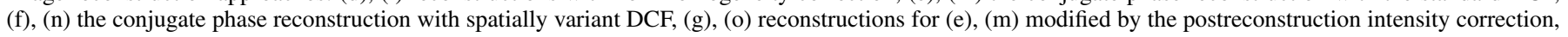
(h), (p) SPHERE reconstruction, and (i)-(k), (q)-(s) iterative reconstruction with 2, 4, and 10 iterations, respectively.

parameters $\mathrm{TR}=3000 \mathrm{~ms}, \mathrm{TE}=15 \mathrm{~ms}$, flip angle $=$ 90 degrees, field of view $=22 \mathrm{~cm}$, slice thickness $=3 \mathrm{~mm}$, $\mathrm{k}$-space diameter 64 , and 3769 samples with $5 \mu$ s sampling for a total readout duration of $18.85 \mathrm{~ms}$. To have a reference image with very little image distortion, an image was acquired using the same pulse sequence parameters, but using an 8-shot acquisition with 495 samples ( $2.48 \mathrm{~ms}$ readout). Field maps were derived using the phase difference method [23] and acquisitions using $\mathrm{TE}=17.5 \mathrm{~ms}$. For the $\mathrm{CP}$ and iterative reconstructions, the 8-shot data were used to derive the field map and were smoothed using the conjugate gradient algorithm with quadratic cost function as previously described. The gradients necessary for spatially variant image reconstructions were calculated from the inhomogeneity map using $3 \times 3$ Sobel derivative kernels. For the SPHERE reconstruction, a single-shot acquisition was used for the field map and it was smoothed using a $3 \times 3$ kernel. We applied the same reconstruction approaches as in the simulation study.

\section{RESULTS}

\section{A. Simulation Study}

Fig. 3 shows simulation results for the circular object with the spiral-out acquisition. The reconstruction using the spatially variant DCF most closely matches the amplitude of the original object (given the limited spatial frequency) while the spatially invariant DCF overestimates the object amplitude. When the postreconstruction intensity correction of (32) is applied, the spatially invariant DCF produces a response very similar to that of the spatially variant DCF, though the spatially variant DCF appears to have a sharper transition. Results were similar for the spiral-in acquisition (not shown) except that the spatially invariant DCF prior to intensity correction underestimated the object intensity. Fig. 3 also shows the effect of errors in the inhomogeneity function used for reconstruction. The largest effect comes from errors in the parallel $\left(g_{x}\right)$ gradient terms, which result in an approximate scaling of the stimulated object due to over- or under-correction of the sample density effects. Errors in the orthogonal $\left(g_{y}\right)$ gradient terms seemed to have no effect on the reconstructed object while errors in $\Delta f$ induced blurring of the object, as expected.

Reconstruction results for the simulated head are given in Fig. 4 for an axial slice approximately $2 \mathrm{~cm}$ above the sphenoid nasal sinus. Fig. 4(a) contains the reference spiral image for the object reconstructed with no magnetic field inhomogeneity. Fig. 4(b) contains the magnetic field map used to generate the simulated data. This map and Fig. 4(c), the gradient map, 

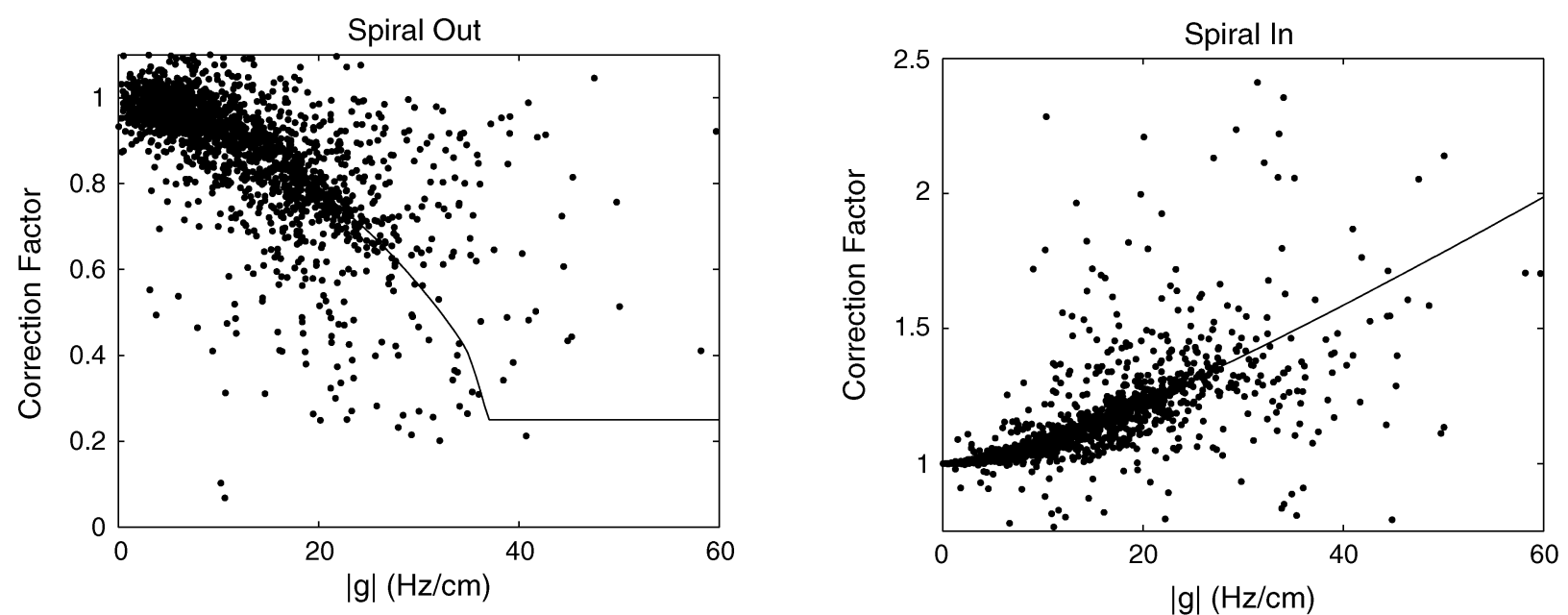

Fig. 5. Comparison of the postreconstruction intensity correction of Equation (32) to corrections provided by full spatially variant DCF for both spiral-out (left) and spiral-in (right) images. Solid line represents the postreconstruction intensity correction factor while dots represent the ratio of pixel intensities for Fig. 4(f) or (e) and Fig. 4(n) or (m), respectively. For gradient echo imaging, there is a reasonably close relationship between these two correction approaches.

TABLE I

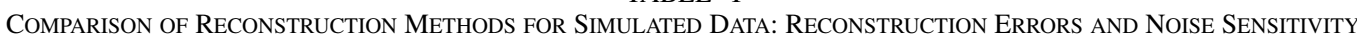

\begin{tabular}{|c|c|c|c|c|}
\hline $\begin{array}{l}\text { Reconstruction } \\
\text { Method }\end{array}$ & $\begin{array}{c}\text { Spiral-Out } \\
\text { Simulation } \\
\text { (no noise) } \\
\% \text { NRMSE }\end{array}$ & $\begin{array}{c}\text { Spiral-In } \\
\text { Simulation } \\
\text { (no noise) } \\
\text { \% NRMSE }\end{array}$ & $\begin{array}{c}\text { Spiral-Out } \\
\text { Simulation } \\
\text { Noise in field map } \\
(\% \text { NRMSE/Hz) }\end{array}$ & $\begin{array}{c}\text { Spiral-Out } \\
\text { Simulation } \\
\text { Noise in data } \\
\text { (\% NRMSE/unit } \\
\text { noise) } \\
\end{array}$ \\
\hline No Correction (a.) & 18.4 & 16.0 & 0 & 1.04 \\
\hline $\begin{array}{l}\text { CP with Invariant } \\
\text { DCF (b.) }\end{array}$ & 16.9 & 10.9 & 0.9 & 1.04 \\
\hline $\begin{array}{l}\text { Slow CP with } \\
\text { Variant DCF (c.) }\end{array}$ & 7.6 & 3.2 & $4.1^{\mathrm{a}} / 1.6^{\mathrm{b}}$ & 1.05 \\
\hline $\begin{array}{l}\text { Fast CP with } \\
\text { Variant DCF (d.) }\end{array}$ & 7.6 & 3.2 & $4.1^{\mathrm{a}} / 1.6^{\mathrm{b}}$ & 1.05 \\
\hline $\begin{array}{l}\text { CP with Intensity } \\
\text { Correction (e.) }\end{array}$ & 9.1 & 4.4 & $10.5^{\mathrm{a}} / 3.1^{\mathrm{b}}$ & 0.92 \\
\hline SPHERE (f.) & 7.3 & 5.5 & 4.4 & 0.99 \\
\hline $\begin{array}{l}\text { Iterative (g.) - } \\
2 \text { Iterations }\end{array}$ & 9.6 & 8.9 & 1.2 & 1.01 \\
\hline $\begin{array}{l}\text { Iterative (g.) - } \\
3 \text { Iterations }\end{array}$ & 7.7 & 7.3 & 1.4 & 1.01 \\
\hline $\begin{array}{l}\text { Iterative (g.) - } \\
4 \text { Iterations }\end{array}$ & 6.6 & 5.4 & 1.7 & 1.00 \\
\hline $\begin{array}{l}\text { Iterative (g.) - } \\
5 \text { Iterations }\end{array}$ & 6.4 & 4.4 & 2.1 & 1.00 \\
\hline $\begin{array}{l}\text { Iterative (g.) - } \\
10 \text { Iterations }\end{array}$ & 6.3 & 3.7 & 2.4 & 1.04 \\
\hline
\end{tabular}

${ }^{\mathrm{a}} 3 \times 3$ and ${ }^{\mathrm{b}} 5 \times 5$ kernels for gradient calculation

were used in the reconstruction process. Fig. 4(d)-(k) shows the eight reconstructed images for the spiral-out data and Fig. $4(1)-(s)$ shows the results for the spiral-in data. Only the results for the fast implementation of the spatially variant DCF [method 4)] are given as they are visually indistinguishable (normalized RMS difference of $\sim 0.1 \%$ ) from the slower implementation [method 3)]. Amongst CP reconstruction methods, the use of the spatially variant DCF and the postreconstruction intensity correction clearly produce images that are closest to the reference image. The image from the SPHERE method and iterative reconstruction also produced images that are very close to the ideal reference image. Table I contains the normalized RMS errors for all reconstruction methods. For the spiral-out data, the iterative method has the lowest NRMSE and the for the spiral-in data, the spatially variant $\mathrm{CP}$ reconstruction had the lowest NRMSE.

Fig. 5 contains a comparison of the pixel values between the spatially invariant and variant DCFs. The postreconstruction intensity correction values are also shown in this figure as a solid line. These plots demonstrate that postreconstruction intensity correction produces images that are similar to those produced by the full spatially variant DCF. This also shows that for gradient echo imaging, a substantial amount of the correction imparted by the variant DCF is an intensity correction.

The noise sensitivity of each of the reconstruction methods is also included in Table I. All of the reconstruction methods pro- 


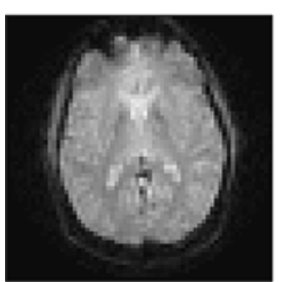

(a)

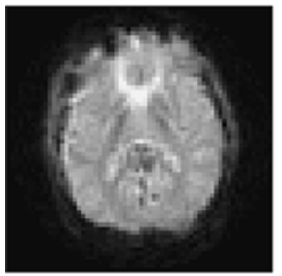

(d)

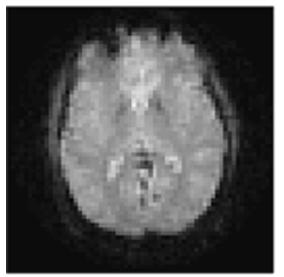

(h)

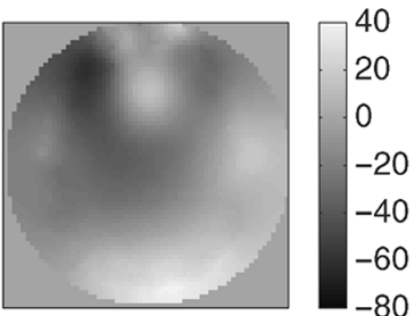

(b)

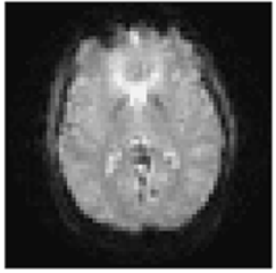

(e)

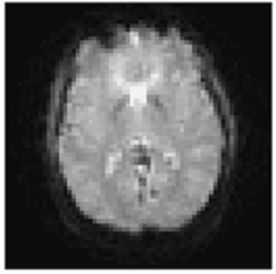

(i)

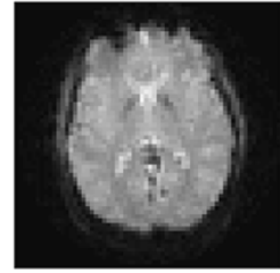

(f)

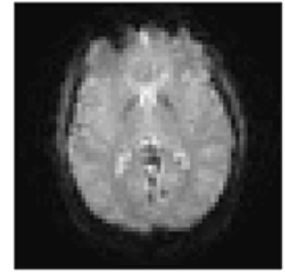

(j)

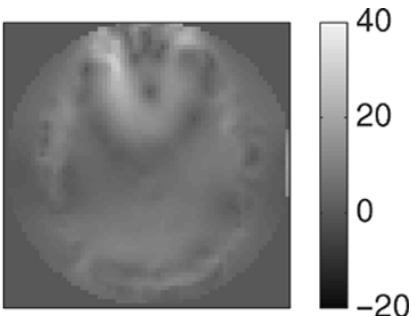

(c)

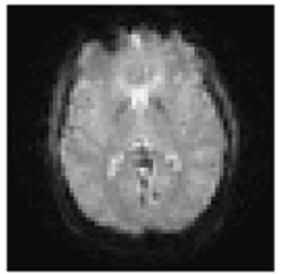

(g)

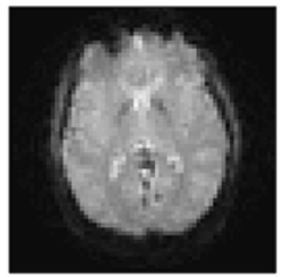

(k)

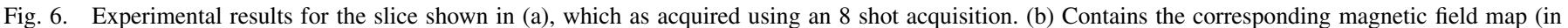

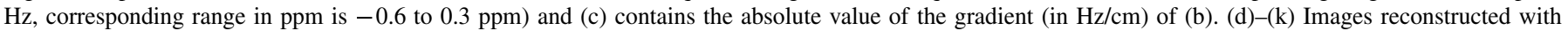

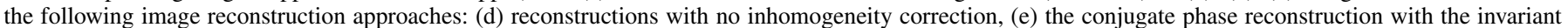

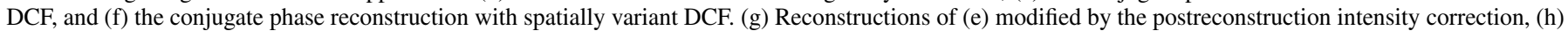
SPHERE reconstruction, (i)-(k) Iterative reconstruction with 2, 4, and 10 iterations, respectively.

duced images with noise measures that varied approximately linearly with respect to noise level in the field map or raw data. The standard CP reconstruction method 2) is least sensitive to noise in the field map and the $\mathrm{CP}$ reconstruction with spatially variant DCF is substantially more sensitive, but this sensitivity can be reduced through the use of larger gradient estimators that employ more averaging. With respect to noise in the raw data, none of the reconstruction methods show any noise amplification relative to the standard Fourier reconstruction. The $\mathrm{CP}$ reconstruction with postreconstruction intensity correction has somewhat reduced noise, most likely from the multiplicative intensity reduction necessary for the spiral-out case.

The computational demands of the different $\mathrm{CP}$ reconstruction methods depend upon the exact implementation. The fast implementation we have used requires precalculation of interpolation coefficients for the gridding step and optimization of the temporal interpolators. On an $800 \mathrm{MHz}$ Intel Pentium processor, this precalculation requires $13.6 \mathrm{~s}$ and if an approximate or generic field map is used [21] most of these calculations can be performed off-line. The additional reconstruction time for each image was $0.6,1.7$, and $0.7 \mathrm{~s}$ for methods 2 ), 4 ), and 5, respectively. These times are for $L=5$ time-segments, and time can be further reduced by reducing the number of time segments, however this increases error. The normalized RMS difference between the fast and slow implementation was about $1.6 \%, 0.5 \%$ and $0.1 \%$ for $L=3,4$, and 5 segments, respectively. The slow implementation method 3 ) of the spatially variant reconstruction requires calculation of a large matrix that implements the density compensation and conjugate phase reconstruction (42.1 s) and a matrix multiplication with the raw data $(1.0 \mathrm{~s})$. Unlike, the fast implementation, the matrix is field map specific and would need to be recalculated for every slice.

\section{B. Experimental Data}

Reconstruction results for the experimental spiral data are given in Fig. 6 for an oblique axial slice approximately $2 \mathrm{~cm}$ above the sphenoid sinus. Fig. 6(a) contains the 8-shot data, which serves as a reference image, Fig. 6(b), (c) shows the magnetic field and gradient maps used in the reconstruction process, and Fig. 6(d)-(k) shows images reconstructed by the various approaches. These results demonstrate that the $\mathrm{CP}$ reconstruction with a spatially variant DCF as well as with intensity correction can produce images that closely approximate the undistorted image acquired using an 8-shot acquisition.

\section{DISCUSSION AND CONCLUSION}

Most image reconstruction in MRI is based on Fourier inversion of k-space data, where k-space is defined from the gradient waveforms. The concept of $\mathrm{k}$-space, however, assumes that the underlying magnetic field is perfectly uniform. When this is not the case, it is appropriate to consider modifications to the k-space formulation that may lead to a modified Fourier 
inversion approach or alternatively, to consider entirely new reconstruction approaches based on modeling the MR physics. In this paper, we consider the former by performing a Taylor series expansion of the underlying magnetic field around every point in the object. Keeping only the first two terms produces a signal equation with an effective k-space trajectory that is distorted and is space variant. Reconstruction from this distorted trajectory requires modification of the sample DCF to a form that is also space variant. Each point in space has its own effective k-space trajectory and thus, its own sample density compensation.

In both simulation and experimental data, we demonstrate the effect of distortions to k-space caused by the gradient term from magnetic field inhomogeneity. In spiral imaging, these distortions can lead to changes in intensity and distortions to the point spread function. A significant portion of the image distortions can be removed by the proposed image reconstruction that uses a spatially variant DCF. In the simulations, however, we found that this method does not lead exactly to the reconstructed image produced by simulation without magnetic field inhomogeneity for two reasons. First, the k-space region acquired is not exactly the same as that acquired for the homogeneous case. For example, in Fig. 2(left), we see a k-space trajectory for a particular point in space that is shifted from the ideal trajectory in Fig. 1. The spiral-in case produces results that are much closer to the homogeneous magnetic field case because the k-space area for the distorted trajectory is more similar to the ideal trajectory. The second reason that the reconstruction is not perfect is that the smoothly varying condition of (23) may be violated for some points, in which case the Jacobian determinant is negative and the Fourier inversion has singularities.

Our derivation of the spatially variant DCF is based on the Jacobian determinant and modifications thereof. For sample values where this determinant is zero, the trajectories cross on top of themselves and the DCF is set to zero. It may, however, be advantageous to keep these samples because they can improve the estimated image in locations with large background gradients. Keeping these sample values, however, may require the use of other approaches for the calculation of DCF. Numerical approaches [6], [7] can accommodate self crossing trajectories gracefully. In addition to spiral acquisitions, these approaches would also be very useful for trajectories that intentionally have self crossings, for example, rosette trajectories [25]. In this case, we are aware of no simple formulae to adjust the DCF for distortions to local k-space. While highly attractive for these cases, the numerical approaches for DCF calculation may be computationally impractical since each image voxel will require a new execution of the DCF calculation procedure.

In addition to application of the spatially variant DCF, we examined an alternate method for correcting for the effects of locally distorted k-space trajectories. In this method, we applied an intensity correction to the images reconstructed using a spatially invariant DCF. The intensity correction is based on the relative density of samples when the true origin of k-space is acquired. This is done for every point in space to create an intensity correction mask. This approach produces results that are very similar to the full spatially variant DCF, as demonstrated by Fig. 4. Fig. 5 further demonstrates that the corrections resulting from the two approaches are very similar, but that differences remain. These differences are likely due to changes in the point spread function (PSF) that result from the density compensation for all parts of $\mathrm{k}$-space rather than just the compensation at the origin. For spin-echo imaging, the origin is sampled with roughly the same density irrespective of background gradients (see top of Fig. 1, for example). In this case, the spatially variant DCF will still improve the PSF, but the postreconstruction intensity correction will have no effect.

We have proposed two approaches for rapid implementation of this reconstruction. The first approach implements the full spatially variant DCF using three image reconstructions and the second does a single reconstruction using a spatially invariant DCF followed by a point-by-point intensity correction. Both approaches use the conjugate phase reconstruction with a time-segmented approximation to the phase accumulation due to off-resonance effects [10], [11]. They also use gridding [8], [9] with FFTs for fast Fourier inversion. The gridding reconstruction with appropriate selection of convolution kernels [9] can produce images with extremely small difference relative to the discrete Fourier summation (NRMSEs of less than $10^{-5}$ ). In addition, the interpolation kernels used to approximate the accumulation of off-resonance phase can be minimized through the use of optimized temporal interpolators, such as the min-max interpolator of Sutton et al. [21]. Both rapid methods required approximately $O\left(L N^{2} \log N\right)$ operations where $N$ is the image dimension and $L$ is the number of time or frequency segments and led to substantial reductions in computation time. The fast approach for the spatially variant DCF [method 4)] was very close to the slower implementation with a normalized RMS difference about $0.1 \%$. This difference included errors resulting from both errors in the fast approximation and differences in how a negative Jacobian determinant was handled.

One apparent disadvantage of the proposed methods is the sensitivity to noise and/or errors in the field and gradient maps. The results in Fig. 3 show that the errors or noise in the gradient component parallel to the true background gradient have predominantly a scaling effect on the image, whereas the errors in the orthogonal direction have negligible effects. Errors in the field map can lead to additional blurring of the image in a manner similar to the standard CP reconstruction. Table I shows that the while the proposed methods do not amplify additive noise in the raw data, they can be quite sensitive to noise in the field map. The relative insensitivity of the standard $\mathrm{CP}$ reconstruction to field map errors together with the reduction in noise sensitivity through the use of a larger kernel for estimating gradients imply that the noise sensitivity is dominated by noise in the gradient maps. In practice, noise in the field maps and gradient maps can be controlled by smoothing the field map and through the use of larger kernels for calculation of gradients. Furthermore, our noise analysis shows that postreconstruction intensity correction is somewhat more sensitive to gradient noise than the reconstruction with the spatially variant DCF. This approach uses the estimated gradient to determine the time of the crossing of the k-space origin in (31) and then uses it again in the determination of the correction factor, resulting in an amplification of the noise relative to the full the spatially variant DCF method.

Our proposed method corrects for the effects of local gradients by adjusting for sample density variations in a local $\mathrm{k}$-space 
representation. There are other approaches that can address the distortions to k-space without explicit calculation of gradient maps and we have tested two of these in the current work. Both of these approaches rely on modeling the signal equation physics [e.g., (15)] with the negative (SPHERE method) or positive (iterative method) field map. Unlike the inverse solution, this equation does not depend on the assumption of a uniform underlying magnetic field. The SPHERE method [18] uses the field map in an attempt to create undistorted k-space data from a distorted image, and the iterative method [21] attempts to find the image that best matches the acquired data using a cost function. Our simulation data summaries in Fig. 4 and Table I show that both of these approaches are effective in reducing both image distortions from field inhomogeneity and intensity variations that result from sample density variations, resulting in reconstruction errors that are similar to those found with spatially variant $\mathrm{CP}$ reconstruction (in some cases better and in other cases worse). The SPHERE method can also be implemented in a computationally efficient manner and can use a distorted field map, but in our implementation it was more sensitive to noise in the field map than the $\mathrm{CP}$ reconstructions. As previously mentioned, with appropriate data weighting, one iteration of the conjugate gradient method is equivalent to the CP reconstruction [21], [24], which does correct for most spatial distortions, but additional iterations (between 3 and 10) were required to eliminate in the intensity variation from the sample density variations. The iterative approach also has fast implementations [21] but with larger numbers of iterations, it is still slower than the proposed approach. The iterative approach could, of course, be initialized with the new CP method and could further reduce the number of iterations. Unlike the CP and SPHERE methods, the iterative reconstruction approach also does not require a smoothly varying field, such as the condition in (23). Finally, the iterative approach also showed some increase in sensitivity to noise in the field map for larger numbers of iterations.

In conclusion, we have developed a new $\mathrm{CP}$ method to reconstruct images corrected for the effects of magnetic field inhomogeneity. Our approach is based on the idea that gradients in the magnetic field inhomogeneity function can lead to spatially varying distortions in an effective $\mathrm{k}$-space trajectory. Since these distortions lead to altered sampling patterns, they require different DCFs for every point in space. We have developed specific formulae for the spatially variant compensation for use in spiral MRI and have described fast implementations of this reconstruction that allow the use of FFTs. We have applied the CP reconstruction with a spatially variant compensation function to simulated and experimental spiral data and this has resulted in substantially more accurate image reconstructions than the standard $\mathrm{CP}$ reconstruction.

\section{REFERENCES}

[1] A. Macovski, "Volumetric NMR imaging with time-varying gradients," Magn. Reson. Med., vol. 2, pp. 29-40, 1985.
[2] S. J. Norton, "Fast magnetic resonance imaging with simultaneously oscillating and rotating field gradients," IEEE Trans. Med. Imag., vol. MI-6, pp. 21-31, 1987.

[3] A. Maeda, K. Sano, and T. Yokoyama, "Reconstruction by weighted correlation for MRI with time-varying gradients," IEEE Trans. Med. Imag., vol. MI-7, pp. 26-32, 1985.

[4] C. Meyer, B. Hu, D. Nishimura, and A. Macovski, "Fast spiral coronary artery imaging," Magn. Reson. Med., vol. 28, pp. 202-213, 1992.

[5] R. D. Hoge, R. K. Kwan, and G. B. Pike, "Density compensation functions for spiral MRI," Magn. Reson. Med., vol. 38, pp. 117-128, 1997.

[6] J. G. Pipe and P. Menon, "Sampling density compensation in MRI: rationale and an iterative numerical solution," Magn. Reson. Med., vol. 41, pp. 179-186, 1999.

[7] V. Rasche, R. Proksa, R. Sinkus, P. Bornert, and H. Eggers, "Resampling of data between arbitrary grids using convolution interpolation," IEEE Trans. Med. Imag., vol. 18, no. 5, pp. 385-392, May 1999.

[8] J. O'Sullivan, "A fast sinc function gridding algorithm for Fourier inversion in computer tomography," IEEE Trans. Med. Imag., vol. MI-4, pp. 200-207, 1985.

[9] J. Jackson, C. Meyer, D. Nishimura, and A. Macovski, "Selection of a convolution function for Fourier inversion using gridding," IEEE Trans. Med. Imaging, vol. 10, no. 3, pp. 473-478, Sep. 1991.

[10] D. C. Noll, C. H. Meyer, J. M. Pauly, D. G. Nishimura, and A. Macovski, "A homogeneity correction method for magnetic resonance imaging with time-varying gradients," IEEE Trans. Med. Imag., vol. 10, no. 4, pp. 629-637, Dec. 1991.

[11] D. Noll, J. Pauly, C. Meyer, D. Nishimura, and A. Macovski, "Deblurring for non-2D Fourier transform magnetic resonance imaging," Magn. Reson. Med., vol. 25, pp. 319-333, 1992.

[12] K. Sekihara, S. Matsui, and H. Kohno, "NMR imaging for magnets with large nonuniformities," IEEE Trans. Med. Imag., vol. MI-4, pp. 193-199, 1985.

[13] P. Jezzard and R. S. Balaban, "Correction for geometric distortion in echo planar images from B0 field variations," Magn. Reson. Med., vol. 34, pp. 65-73, 1995.

[14] R. Deichmann, O. Josephs, C. Hutton, D. R. Corfield, and R. Turner, "Compensation of susceptibility-induced BOLD sensitivity losses in echo-planar fMRI imaging," Neuroimage, vol. 15, pp. 120-135, 2002.

[15] Y. Yang, H. Gu, W. Zhan, S. Xu, D. A. Silbersweig, and E. Stern, "Simultaneous perfusion and BOLD imaging using reverse spiral scanning at 3T: characterization of functional contrast and susceptibility artifacts," Magn. Reson. Med., vol. 48, pp. 278-289, 2002.

[16] D. Noll, "Rapid MR image acquisition in the presence of background gradients," presented at the 2002 IEEE Int. Symp. Biomedical Imaging, Washington, DC, 2002.

[17] P. J. Reber, E. C. Wong, R. B. Buxton, and L. R. Frank, "Correction of off resonance-related distortion in echo-planar imaging using EPI-based field maps," Magn. Reson. Med., vol. 39, pp. 328-330, 1998.

[18] Y. M. Kadah and X. Hu, "Simulated phase evolution rewinding (SPHERE): a technique for reducing B0 inhomogeneity effects in MR images," Magn. Reson. Med., vol. 38, pp. 615-627, 1997.

[19] P. Irarrazabal, C. H. Meyer, D. G. Nishmura, and A. Macovski, "Inhomogeneity correction using an estimated linear field map," Magn. Reson. Med., vol. 35, pp. 278-282, 1996.

[20] H. Schomberg, "Off-resonance correction of MR images," IEEE Trans. Med. Imag., vol. 18, no. 6, pp. 481-495, Jun. 1999.

[21] B. P. Sutton, D. C. Noll, and J. A. Fessler, "Fast, iterative image reconstruction for MRI in the presence of field inhomogeneities," IEEE Trans. Med. Imag., vol. 22, no. 2, pp. 178-188, Feb. 2003.

[22] P. Bornert, B. Aldefeld, and H. Eggers, "Reversed spiral MR imaging," Magn. Reson. Med., vol. 44, pp. 479-484, 2000.

[23] E. Schneider and G. Glover, "Rapid in vivo proton shimming," Magn. Reson. Med., vol. 18, pp. 335-347, 1991.

[24] Y. Kadah and X. Hu, "Algebraic reconstruction for magnetic resonance imaging under $\mathrm{B}_{0}$ inhomogeneity," IEEE Trans. Med. Imag., vol. 17, no. 3, pp. 362-370, Jun. 1998.

[25] D. C. Noll, "Multishot rosette trajectories for spectrally selective MR imaging," IEEE Trans. Med. Imag., vol. 16, no. 4, pp. 372-377, Aug. 1997. 